\title{
STATUS OF THE PROJECT OF RADIOISOTOPE COMPLEX RIC-80 (RADIOISOTOPES AT CYCLOTRON C-80) AT PNPI*
}

\author{
V.N. Panteleev ${ }^{* *}$, A.E. Barzakh, L.Kh. Batist, D.V. Fedorov, A.M. Filatova, \\ V.S. Ivanov, F.V. Moroz, P.L. Molkanov, S.Yu. Orlov, Yu.M. Volkov
}

NRC “Kurchatov Institute” PNPI, Gatchina, Leningrad District, Russia

\begin{abstract}
Presently, the cyclotrons are playing a very important role in the production of radionuclides for medicine, which are very safe and reliable installations. From radioisotopes produced with cyclotrons, it is rather easy to select one that has appropriate nuclear-physical characteristics for medical use, in comparison to the ones produced with reactors. At PNPI, a high current cyclotron C-8o with the energy of extracted proton beam of 4O-8o $\mathrm{MeV}$ and the current up to $200 \mu \mathrm{A}$ is under construction. One of the main goals of $\mathrm{C}-8 \mathrm{O}$ is the production of a wide spectrum of medical radionuclides for diagnostics and therapy. At present time, a project is worked out for the construction of radioisotope complex $R I C-80$ (Radioisotopes at the cyclotron $C$-8o) at the beam of C-8o. In the presented submission, the project of RIC-80 complex is discussed, which includes three target stations for the production of a large set of radionuclides for medicine. The peculiarity of the proposed radioisotope facility is the use of the mass-separator with the target-ion source device as one of the target stations for on-line, or semi on-line production of a high purity separated radioisotopes. The first results on the target development for production of different medical radionuclides, including radioisotope generator for PET diagnostics ${ }^{8}{ }^{2} \mathrm{Sr}$, are presented as well.
\end{abstract}

Key words: Medical radionuclides, radioisotope complex, production targets, mass-separator, radioisotopes of a high purity

DOI: 10.21175/RadJ.2016.02.017

\section{INTRODUCTION}

It is well known that the main sources for production of the radioactive nuclides are thermal neutrons reactors. Although in the last decades for that purpose on the front place go the accelerators of the charged particles - cyclotrons, as the most safe and reliable technological installations. The list of cyclotron radio nuclides is longer and more varied, than ones obtained with the reactors. One of the most important characteristics of cyclotron produced radionuclides is emission of positrons that allows using them for the PET (Positron Emission Tomography) diagnostics. The rapidly growing nuclear physics studies, appropriate experimental method developments and use of very sensitive and effective detectors has given a very good possibility for elaboration of completely new direction in medical diagnostic and therapy of different kind diseases which is well known presently as nuclear medicine. The exceptional ability of the nuclear medicine methods consists in the possibility to detect and treat a large set of different diseases at a very early stage, saving many human lives and high money expenses for the treatment. The technology of nuclear medicine used for diagnostic and therapy as well is based on the employment of by a special way produced radioactive isotopes with selected properties. The most widely used methods in the nuclear diagnostic are the methods, when the used pharmaceutics are marked by specially selected radio nuclides. Measuring by specially designed detector devices the distribution of the inserted into a human body radio isotopes it is possible to observe the function of investigated organs or their parts. Similar ways are used for the treatment of a different kind of malignant tumors. One of the main advantages of the nuclear radio medicine methods is that the diagnostic and treatment can be carried out at a very early stage of the disease.

This work is the first part of the program on the target development for the new project RIC-80 (Radioactive Isotopes at cyclotron C-80) [1], which is being carried out presently in PNPI. The main task of this work is the method developments on the production and separation of isotope-generator ${ }^{82} \mathrm{Sr}$ which is used for PET diagnostics of hart diseases and also for some other isotopes utilized for diagnostics and therapy. The methods have been worked out making use of the proton beam of a $1 \mathrm{GeV}$ synchrocyclotron with the purpose to use the developed target prototypes at the new radio isotope installation RIC-80 with the proton energy $80 \mathrm{MeV}$ and proton beam intensity up to $200 \mathrm{MeV}$.

2. SCHEME OF THE DESIGNED INSTALLATION OF RIC-80 (RADIOACTIVE ISOTOPES ON CYCLOTRON C-80)

In fig.1 the layout of RIC-80 installation is presented. It intended for production of medical radio-

\footnotetext{
* The paper was presented at the Third International Conference on Radiation and Applications in Various Fields of Research (RAD 2015), Budva, Montenegro, 2015.
}

**vnp@pnpi.spb.ru 
nuclides in the beam of the C-8o cyclotron, which is under construction at PNPI in Gatchina (Russia). The proton beam energy at the target can be varied in the interval 40-80 MeV. The proton beam intensity will be up to $200 \mu \mathrm{A}$. This cyclotron is intended for treatment of ophthalmologic diseases by irradiations of a malignant eye formation, as well as for production of a wide spectrum of medical radionuclides for diagnostics and therapy. The cyclotron is located in the right side of experimental hall (ground floor) of the PNPI $1 \mathrm{GeV}$ synchrocyclotron.

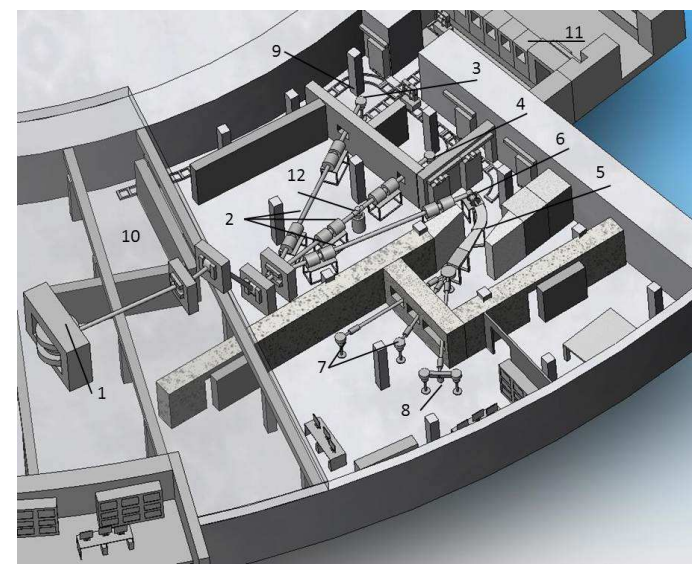

Figure 1. Layout of RIC-80 radioisotope complex. 1cyclotron C-8o, 2 - proton beam tubes, 3 - target station for radiochemical extraction of produced isotopes, 4 - target station for dry (high temperature heating) extraction of produced isotopes, 5 - mass-separator for production of medical isotopes of a high purity, 6 - target station of massseparator, 7 - collecting stations for separated radio nuclides, 8 - detector stations for obtained radio nuclide purity measurement, 9 - transportation system for activated targets to special storage places or to hot cells, 10 -storage place for irradiated targets, 11 - hot cells, 12 - pneumatic rabbit station.

At the drawing the deflecting and focusing magnet elements are shown, which are installed on the move of the proton beam to target stations and to the massesseparator target. Two available target stations allow us to get higher efficiency in the proton beam use, since during the radiation cooling of the irradiated target, where a long lived radionuclide was produced, another target can be irradiated to produce a different radionuclide. The mass-separator with its target station will allow to get the separated medical radio nuclides of a high purity, which will be implanted into a corresponding collectors from those can be easily extracted. Target stations will be equipped with special devices to download highly radioactive targets into protection containers to transport them safely to special storage places, or to hot cells for the aftertreatment and production of corresponding preparations for pharmaceutics.

\subsection{Estimated yields of radio nuclides to be produced at RIC-80 facility.}

C-8o facility will be the largest in Russia cyclotron facility in respect of beam energy accumulated in production of radionuclides and providing enough high energy of bombarding particles (proton energy of 80
$\mathrm{MeV})$. It gives a possibility to produce sources of a high activity within practically the whole list of radio nuclides produced at accelerators. In table 1 estimated yields of radio nuclides are shown, which are planned to be obtained at RIC-8o facility.

Table 1. Activity in target of radionuclides planned to be obtained at RIC-80 facility with proton beam energy of 40$80 \mathrm{MeV}$ and current of $100 \mathrm{uA}$.

\begin{tabular}{|l|l|c|c|c|}
\hline Isotope & Half-life & Target & $\begin{array}{l}\text { Irradiation } \\
\text { time (h) }\end{array}$ & $\begin{array}{l}\text { Target } \\
\text { activity } \\
\text { (Ci) }\end{array}$ \\
\hline Ge-68 & $270.8 \mathrm{~d}$ & $\mathrm{Ga}$ & 240 & 2 \\
\hline Sr-82 & $25.55 \mathrm{~d}$ & $\mathrm{RbCl}, \mathrm{Rb}$ & 240 & 12,15 \\
\hline Mo-99 & $2.74 \mathrm{~d}$ & $\mathrm{Mo}$ & 240 & 8 \\
\hline In-111 & $2.8 \mathrm{~d}$ & $\mathrm{Cd}$ & 25 & 25 \\
\hline I-123 & $13.27 \mathrm{~h}$ & $\mathrm{Te}$ & 5 & 11 \\
\hline I-124 & $4.17 \mathrm{~d}$ & $\mathrm{Te}$ & 25 & 10 \\
\hline Tb-149 & $4.1 \mathrm{~h}$ & $\mathrm{Gd}$ & 12 & 3 \\
\hline Ra-223 & $11.4 \mathrm{~d}$ & ThC & 240 & 7 \\
\hline
\end{tabular}

As one can see from the table, RIC-80 facility will provide the production of a large amount of radionuclides produced at present by this kind of installations. It is necessary to emphasize that the activities of radioisotopes are shown for production in the target. The actual activities extracted out of target material can be less because of incomplete extraction of produced radioisotopes. For the improvement of extraction efficiencies for targets under development the test experiments with target prototypes are performed at the synchrocyclotron in operation at PNPI for effective production of listed nuclides. Possibilities of the radionuclides production at RIC-80 installation are not exhausted by the list of isotopes provided in Table 1 . The unique parameters of the C-80 cyclotron will allow producing the broad nomenclature of radioisotopes, which are at present under discussion in corresponding publications as very perspective for diagnostics and therapy. They are $\mathrm{Cu}-64, \mathrm{Cu}-67, \mathrm{Ga}-67$, Br-77, Rb-81, At-211.

The comparison of used for many years isotopes with new isotopes, which are under discussion and used for clinical tests brings to a conclusion on trends to use more short-lived radionuclides to aim the diagnostics and therapy that significantly reduces the radiation rate for patients.

\subsection{On-line mass-separator at RIC-8o installation to obtain medical isotope beams of the high purity}

The production and study of radioactive isotopes by ISOL systems (Isotope Separator On-Line) is one of the fundamental directions of nuclear physics, as well as study of astrophysical processes in laboratory conditions. The main problem - the extraction and isotopic separation produced radio nuclides is under successful solution for almost 50 years already by means of ISOL installations at beams of different bombarding particles - protons, neutrons and heavy ions. Such on-line systems like ISOLDE (CERN, Switzerland), ISAC (TRIUMF, Canada), IRIS (PNPI, Gatchina) $[2,3,4]$ operation on-line at proton beams, allows to obtain at present more than 2000 separated isotopes of almost all elements of the Periodic system. 
Produced radioactive isotopes with half-life from several milliseconds to several thousand years are used in nuclear physics study, in study of solid state physics and in the radiation medicine.

In a very short way the work of an ISOL facility can be described as follows. The beam of bombarding particles (see fig. 1) is directed to the target of the mass-separator, which is located inside the vacuum chamber at the high temperature (of $1800-2200^{\circ} \mathrm{C}$ ), where radionuclides are produced as a result of nuclear reactions. The products formed inside a target material get out of target container into an ion source, where the ionization occurs. Ions, going out of ionizer, are formed by the field of extraction electrode into a beam of $\sim 2 \mathrm{x}$ 10-2 radians divergence. Going through a focusing lens, ion beam is converted into a parallel and gains the energy of $30-50 \mathrm{keV}$. Later the beam of mono energy ions enters the magnet-analyzer, where the mass separation occurs. The cross-section dimensions of the beam at the magnet focal plane of the mass- separator are of about $1 \mathrm{~mm}$ in vertical and $1.5 \mathrm{~mm}$ in horizontal directions. Separated according their masses beams of radioactive ions enter the switchyard chamber and after that they go along vacuum ion guide tubes to the experimental hall, where they are implanted into corresponding collectors. Sources of radio nuclides obtained without any carrier and admixtures are measured in time of the accumulation process for the determination of their purity and activity rate by means of $\alpha, \beta$ and $\gamma$ detectors installed close to collectors, where the implantation of radioactive ion beams occurs. Coming from aforesaid one, the massseparator method possesses the following values:

- allows to obtain very pure beams of medical isotopes of many elements;

- several separated radionuclides can be accumulated simultaneously;

- depth implantation of several times of ten Å allows to use very fine organic substrates that significantly simplifies the production of medical preparations;

- the method of the radioactive ions implantation allows to obtain the unique generators of radioactive noble gases;

- the target is used many times and does not require reconstruction;

- except a number of relatively long-lived nuclides, mass-separator on-line at accelerator or reactor allows to obtain a lot of new "short-lived" radioactive isotopes $\left(T_{1 / 2}=\right.$ from several tens of minutes to several hours) for diagnostics;

- process of the diagnostics can be realized "on-line" in medical laboratories, which are based on installations produced a big variety of short-lived mass-separated radioactive nuclides of a high purity.

As the isotope production efficiency by the massseparator method is dependent on the ionization efficiency of produced nuclide and the ion source used, it is necessary, calculating the expected radioactivity, to take into account this value for the nuclide being produced. As to radionuclides listed above the ionization efficiency $\varepsilon_{\mathrm{i}}$ may be the following: for $\mathrm{Rb}-\varepsilon_{i}$ $\approx 80 \%$; for $\mathrm{Sr}, \mathrm{In}, \mathrm{Ra}-\varepsilon_{i} \approx 20-40 \%$; for I - $\varepsilon_{i} \approx 30 \%$.

\section{TARGET DEVELOPMENT FOR THE RIC-8O PROJECT}

\subsection{Experiment description and experimental results on ${ }^{82} \mathrm{Sr}$ production}

For the first experimental tests the niobium foils, pills of yttrium carbide and rubidium chlorine powder were used as target materials for production of ${ }^{82} \mathrm{Sr}$. ${ }^{82} \mathrm{Sr}$ with a half-live $T_{1 / 2}=25.55 \mathrm{~d}$ is a generator for its daughter isotope ${ }^{82} \mathrm{Rb}\left(T_{1 / 2}=1.25 \mathrm{~min}\right)$ which is widely used in PET diagnostics. The irradiated target material was placed into Ta-W container which was designed as a prototype of a target device for production of ${ }^{82} \mathrm{Sr}$ at RIC-80. In a high vacuum the target material was heated to separate the produced nuclides and the target material [1].

For the target heating tests the target-prototype was manufactured with parameters close to the working parameters of the target which will be constructed for the RIC-80 facility. The targetprototype parameters were the following: the length of the target container $-200 \mathrm{~mm}$; the target container diameter $-30 \mathrm{~mm}$. The target-prototype prepared for the subsequent irradiation at the proton beam to accumulate ${ }^{82} \mathrm{Sr}$ in the target material is shown in fig. 2.

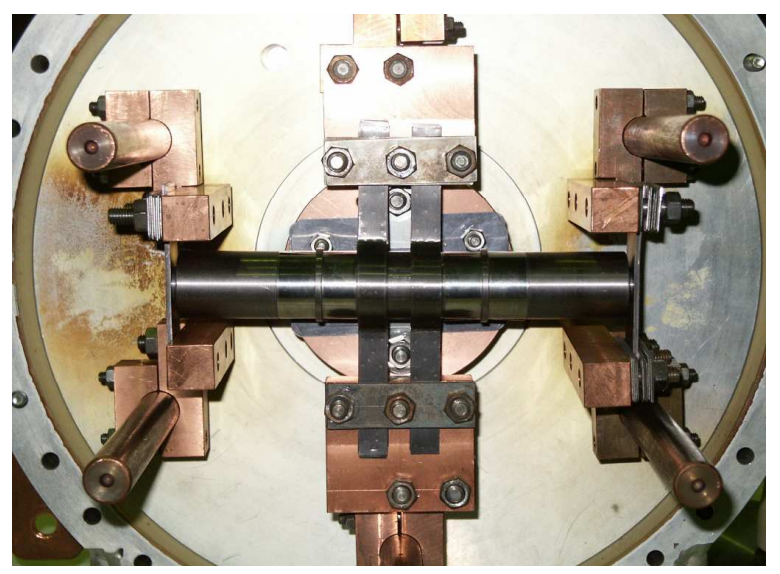

Figure 2. The target-prototype specially constructed for the tests on the production and thermal extraction of generatorisotope ${ }^{82} \mathrm{Sr}$ and other radionuclides from irradiated target materials.

The samples of different target materials after irradiation by the $1 \mathrm{GeV}$ proton beam were placed into the target container (tungsten-tantalum cylinder). In the central part the target container was connected to the tungsten tube with a hole of $2 \mathrm{~mm}$ in diameter. Through this tube the radioactive nuclides accumulated in the target material were evaporated in the course of target thermal heating and absorbed by the tantalum foil collector, contacting with cooper flange, cooled by floating water. For the tungsten tube, which the evaporated nuclides passed through, the same method of the resistant heating was used as for the target container as well. The target was heated to 
its working temperature $2000-2100^{\circ} \mathrm{C}$. The high vacuum test bench was specially constructed for testing high temperature targets with the heating power up to $\sim 9 \mathrm{~kW}$. The target developed construction allows to produce and select radionuclides from different target materials such as refractory metal foils, refractory metal carbides, liquid metals and metal powder compounds as well. There were some stages in the process of the target heating in a high vacuum for extraction of produced in the target material radioactive species. To select strontium from yttrium carbide target, which release from the target is characterized by relatively slow process diffusion and effusion, the target heating was started at low temperatures 1500-1800 to evaporate radioactive species with faster diffusion-effusion properties, such as $\mathrm{Rb}, \mathrm{Mn}$ and other radio nuclides with high values of diffusion-effusion parameters. After that, strontium may be selectively extracted by heating the target with a higher temperature. In fig. 3 gamma spectrum is presented of irradiated sample of yttrium carbide measured by the hyperfine germanium $\gamma$-detector before the target heating. In fig. 4 gamma spectrum is presented of collected radioactivity at the cooled collector during the heating of irradiated yttrium carbide sample.

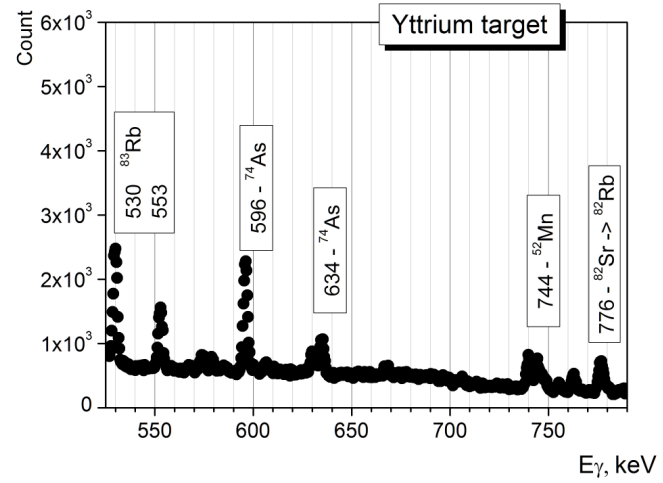

Figure 3. Gamma-spectrum of irradiated sample of yttrium carbide. Gamma-line $776 \mathrm{keV}$ belongs to decay of ${ }^{82} \mathrm{Sr}$ daughter isotope ${ }^{82} \mathrm{Rb}$, used for PET diagnostics.

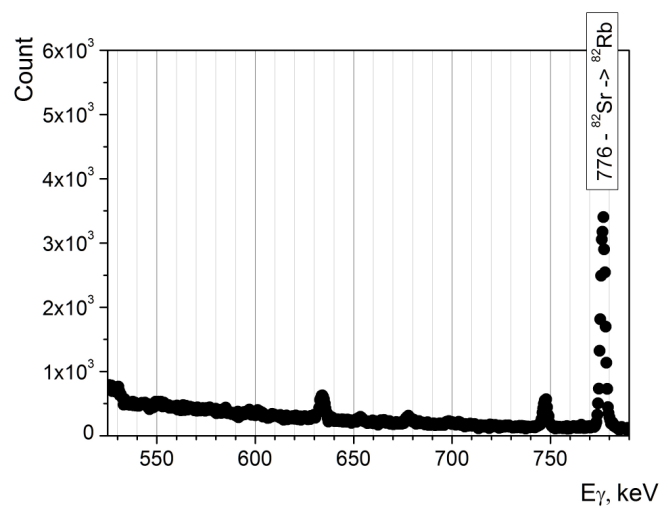

Figure 4. Gamma spectrum of collected radioactivity at the cooled collector during the heating of irradiated yttrium carbide samples at a temperature interval $1950-2000^{\circ} \mathrm{C}$.
As one can see from fig. 3, 4 finally almost all radioactive species were evaporated from the irradiated target, except strontium. The final efficiency of extraction of strontium after the yttrium carbide heating at the temperature $1950-2000^{\circ} \mathrm{C}$ during the time interval of 10 hours was about $90 \%$. The efficiency of removing collected radioactive strontium from the tantalum collector by $\mathrm{HCl}$ of $20 \%$ concentration was $85 \%$. The yttrium carbide target is intended to be used for ${ }^{82} \mathrm{Sr}$ production by a mass-separator method. This target material possesses good characteristics (melting point, boiling point) to be used as a target under a high temperature coupled with the mass-separator ion source of surface ionization.

For production of ${ }^{82} \mathrm{Sr}$ by a dry, high temperature heating method traditional target materials rubidium chloride and metallic rubidium have been used. For this purpose irradiated powder of $\mathrm{RbCl}$ or metallic rubidium in a tantalum cavity was placed to a heated target container. All manipulations with metallic rubidium were carried out in argon atmosphere. In a high vacuum the target material during target heating at a temperature lower than $1000^{\circ} \mathrm{C}$ was evaporated and collected in a special cavity for the target material collection. All target material was evaporated for the time of some hours. After that the cavity was exchanged by a tantalum foil collector for collection of strontium isotopes, which were escaping from the target container at a temperature of $1900-1950^{\circ} \mathrm{C}$. For the heating time of 2 hours $90 \%$ of strontium was evaporated and collected at the cooled tantalum collector. In fig. 5 part of measured $\gamma$-spectrum of $\mathrm{RbCl}$ sample is shown before the heating (black circles) and collected strontium-82 during the heating irradiated rubidium chloride with the temperature $1900-1950^{\circ} \mathrm{C}$ after evaporation of the target material (open circles).

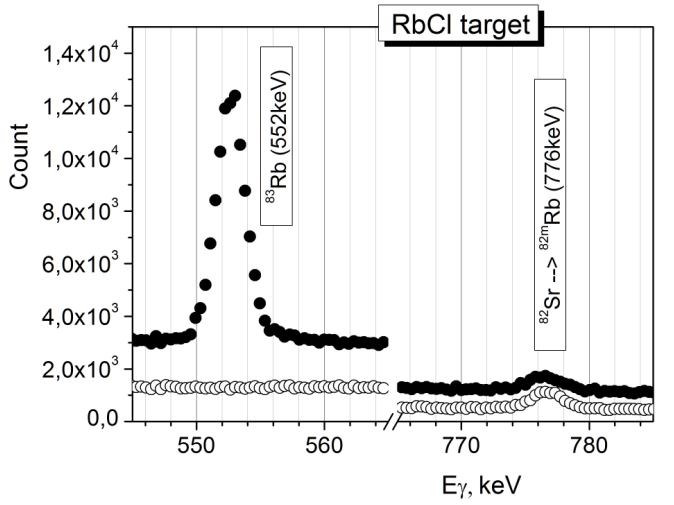

Figure 5. Part of measured $\gamma$-spectrum of $\mathrm{RbCl}$ sample before the heating and collected strontium-82 during the heating target cavity with the temperature $1900-1950^{\circ} \mathrm{C}$ after evaporation of the target material.

\subsection{Production of $\mathrm{Tl}$ isotopes from a led target}

For diagnostics of myocardial diseases in nuclear medicine radionuclide ${ }^{201} \mathrm{Tl}\left(T_{1 / 2}=3.04 \mathrm{~d}\right)$ is used. ${ }^{201} \mathrm{Tl}$ can be effectively produced from the led target of natural or enriched abundance. The production crosssections are very high and this method could be used for effective production of ${ }^{201 \mathrm{~T}}$. The only problem is 
the admixture of neighboring isotopes with close halflives ${ }^{200} \mathrm{Tl}\left(T_{1 / 2}=1.09 \mathrm{~d}\right)$ and ${ }^{202} \mathrm{Tl}\left(T_{1 / 2}=12.23 \mathrm{~d}\right)$. In this case a mass-separator method of production of a high purity mass-separated ${ }^{201} \mathrm{Tl}$ can be explored.

In the experiment on the thallium isotope production-extraction a target of led of a natural abundance was irradiated at the $1 \mathrm{GeV}$ proton beam of PNPI synchrocyclotron. The irradiation time was 24 hours. After 10 days of the radiated led cooling it was transported to the experimental hall of the IRIS facility and was placed into the heating tungsten container in the vacuum test bench. The gamma spectrum of irradiated led sample before its heating is shown in fig. 6 .

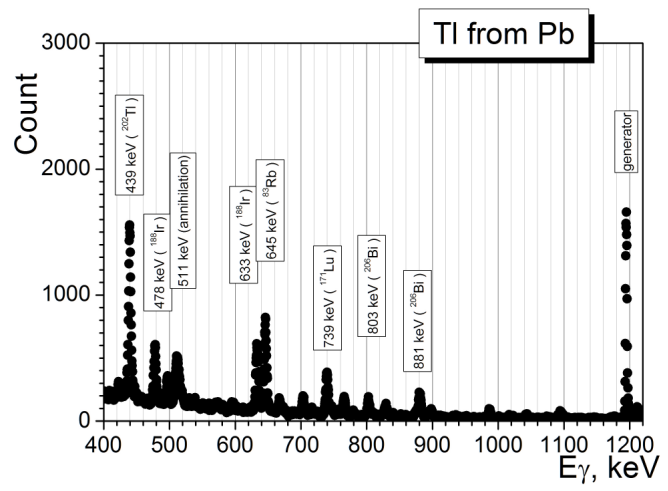

Figure 6. Gamma-spectrum of irradiated led sample of natural abundance after 10 days of radiation cooling. The gamma line $439 \mathrm{keV}$ is from decay of ${ }^{202} \mathrm{Tl}\left(\mathrm{T}_{1 / 2}=12.23 \mathrm{~d}\right)$.

The main goal of the first experiment on $\mathrm{Tl}$ isotope production from a led target was to find the heating conditions for a selective $\mathrm{Tl}$ extraction from the irradiated led sample. It was expected that for thallium, which is a very easy volatile element, speed of evaporation from a melted led target material will be faster than for other radioactive species. As the value of the $\mathrm{Pb}$ melting point is $327^{\circ} \mathrm{C}$, the $\mathrm{Tl}$ extraction has been carried out at the temperature of about $400^{\circ} \mathrm{C}$ to be sure that the led sample was completely melted. In fig. 7 the gamma spectrum of species evaporated at $400^{\circ} \mathrm{C}$ from the led sample on the cooled tantalum collector is shown.

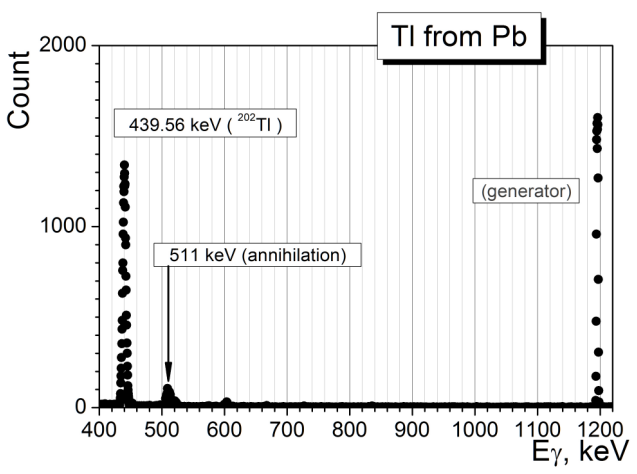

Figure 7. Gamma spectrum of species evaporated at $400^{\circ} \mathrm{C}$ from the led sample and condensed on the cooled tantalum collector. The time of evaporation was 2 hours.
As we can see from the comparison of gamma spectra from figs. 6 and 7, thallium selective evaporation from a melted led target is very high. For two hours of heating about $80 \%$ of ${ }^{202} \mathrm{Tl}$ were escaped from the target and absorbed by the collector. In this case situation is completely different to one with the strontium extraction, when the target material (niobium foils, or yttrium carbide for RIC-80) should be heated up to $2000^{\circ} \mathrm{C}$.

\subsection{Production of alpha decaying radium isotopes from a uranium carbide target}

As it was established, the radio nuclides decaying by alpha particle emission can be a very effective tool for therapy of different malignant tumors at a very early stage of their formation. The main advantage of alpha particle use (so called "alpha-knife") is their very short range (60-80 microns) in a biological tissue. It is very important for the treatment of a small malignant formation. The action of a radionuclide, decaying by alpha particles is very effective, as it is very local and does not destroy the surrounding well tissues. Among alpha decaying radio nuclides which have been tested and can be used for therapy there are two isotopes ${ }^{223} \mathrm{Ra}\left(T_{1 / 2}=11.4 \mathrm{~d}\right)$ and ${ }^{224} \mathrm{Ra}\left(T_{1 / 2}=3.66 \mathrm{~d}\right)$, which can be rather effectively produced by proton irradiation of uranium or thorium targets. In fig. 8 the cross-sections of ${ }^{227}$ Th from the thorium target [5] is presented.

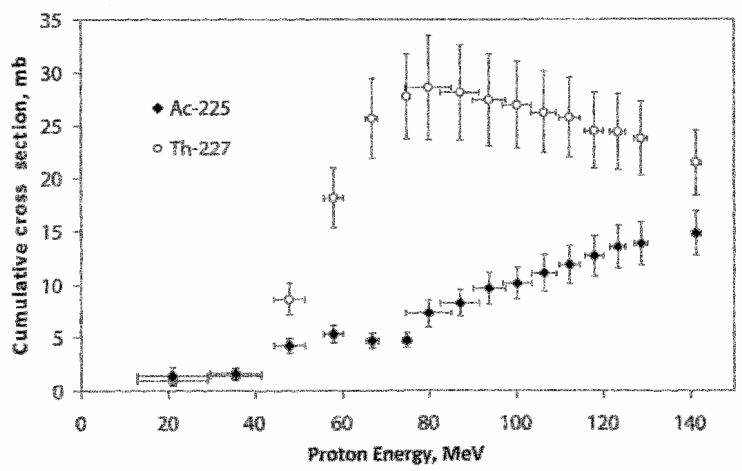

Figure 8. Cross-sections of 227Th production from the thorium target versus the energy of impinging protons. By open circles the cross-sections of $227 \mathrm{Th}$ production is shown [5].

As it seen from fig.8, ${ }^{227} \mathrm{Th}$ has a rather high value of production cross-section by spallation reaction from natural ${ }^{232} \mathrm{Th}(100 \%)$ target at the proton energy close to $80 \mathrm{MeV}$. ${ }^{223} \mathrm{Ra}$ is produced in the subsequent alpha decay of $227 \mathrm{Th}$ :

$$
\begin{aligned}
& { }^{227} \mathrm{Th}(18.7 \mathrm{~d})-{ }^{223} \mathrm{Ra}(11.4 \mathrm{~d})-{ }^{219} \mathrm{Rn}(3.96 \mathrm{~s})- \\
& { }^{215} \mathrm{Po}(1.8 \mathrm{~ms})-{ }^{211} \mathrm{~Pb}(36.1 \mathrm{~min})-{ }^{211} \mathrm{Bi}(2.14 \mathrm{~min})- \\
& { }^{207 T l}(4.77) \mathrm{min}-{ }^{207} \mathrm{~Pb}(\text { stable })
\end{aligned}
$$

As it is seen from the decay chain presented above, the decay of selected ${ }^{223} \mathrm{Ra}$ gives three alpha particles, that considerably increases the method efficiency.

In our experiments we used ${ }^{238} \mathrm{U}$ target (uranium mono carbide of a high density [6]) for production and a high temperature extraction of radium isotopes, decaying by alpha particle emission. The uranium carbide target of a density $11 \mathrm{~g} / \mathrm{cm}^{3}$ was irradiated for 24 hours by the proton beam of PNPI synchrocyclotron 
with the intensity of $0.1 \mu \mathrm{A}$. The target mass was about 1g. After one month of radiation cooling the target was placed into the heating tungsten container in the vacuum test bench. The radium isotope extraction has been carried out at the temperature of about $2400^{\circ} \mathrm{C}$, which is close to the melting point $\left(2500^{\circ} \mathrm{C}\right)$ of uranium mono carbide target material. In fig. 9 the alpha spectrum of species evaporated at $2400^{\circ} \mathrm{C}$ from a UC target on the cooled tantalum collector is shown.

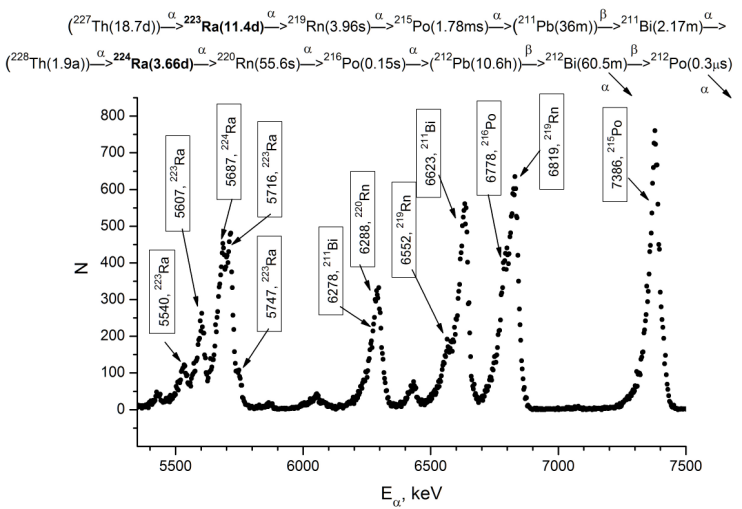

Figure 9. The alpha spectrum of species evaporated at $2400^{\circ} \mathrm{C}$ from a UC target on the cooled tantalum collector.

We have measured alpha particles also from the decay of a long-lived ${ }^{228} \mathrm{Th}\left(T_{1 / 2}=1.9 \mathrm{y}\right)$. In the fig. 9 we can see the chain of appropriate alpha lines:

$$
\begin{array}{llcl}
{ }^{228} \mathrm{Th}\left(\mathrm{T}_{1 / 2}=1.9 \mathrm{y}\right) & - & { }^{224} \mathrm{Ra}\left(\mathrm{T}_{1 / 2}=3.66 \mathrm{~d}\right) & - \\
{ }^{22} \mathrm{Rn}\left(\mathrm{T}_{1 / 2}=56 \mathrm{~s}\right) & - & { }^{216} \mathrm{Po}\left(\mathrm{T}_{1 / 2}=145 \mathrm{~ms}\right) & - \\
{ }^{212} \mathrm{~Pb}\left(\mathrm{~T}_{1 / 2}=10.6 \mathrm{~h}\right) & -\quad{ }^{212} \mathrm{Bi} \quad\left(\mathrm{T}_{1 / 2}=25 \mathrm{~min}\right) & - \\
{ }^{208} \mathrm{Tl}\left(\mathrm{T}_{1 / 2}=3 \mathrm{~min}\right) & { }^{208} \mathrm{~Pb}(\text { stable }) .
\end{array}
$$

The experiment on the ${ }^{223,224} \mathrm{Ra}$ production has demonstrated a principal possibility to obtain alpha decaying radium radionuclides from a high density UC target. There is a possibility to increase the yields of Ra isotopes more than order of magnitude, using the ThC target instead of UC one. The technology of the ThC target has been already developed. This target material possesses good characteristics (melting point, boiling point) to be used as a target under a high temperature coupled with the mass-separator ion source of surface ionization. It is planned to test this target material for production of radium and other alpha decaying isotopes for medicine in 2015 .

\section{CONCLUSION}

At PNPI a high current cyclotron C-8o with the energy of extracted proton beam of $40-80 \mathrm{MeV}$ and the current up to $200 \mu \mathrm{A}$ will be put into operation in the second part of 2015. One of the main goals of C-8o is production of a big number of medical radio nuclides for diagnostics and therapy. At present time the construction of radioisotope complex RIC-80 (Radio Isotopes at the cyclotron $\mathrm{C}-80$ ) at the beam of $\mathrm{C}-8 \mathrm{O}$ is carried out. The peculiarity of the proposed radioisotope facility is the use of the mass-separator with the target-ion source device as one of the target stations for on-line, or semi on-line production of a high purity separated radioisotopes.

The important part of the work was devoted to the target development for the new project RIC-80. For the tests carried out different kinds of target materials were explored (foil of a refractory metal, metals, high density metal carbide and metal salt powder). The tested target materials will be used for manufacture real target prototypes for PNPI radioisotope complex. Investigated target materials can be used as well for the mass-separator target construction of the massseparator station for production at RIC-80 radioisotopic medical beams of a very high purity. The following stage of the work will be the target unit prototype construction with the amount of the target material (40-60 grams) which is required for medical radionuclide production at the RIC-80.

\section{REFERENCES}

1. High Energy Physics Division. Main scientific Activities, V.N. Panteleev et al., National Research Center "Kurchatov Institute" B.P. Konstantinov Petersburg Nuclear Physics Institute, Gatchina 2013.

2. D. Voulot et al., "Radioactive beams at REX-ISOLDE: Present status and latest developments," Nucl. Instr. and Meth. B, vol. 266, pp. 4103-4107, Jun. 2008.

3. M. Dombsky et al., "High intensity targets for ISOL, historical and practical perspectives," Nucl. Instr. and Meth. B, vol. 266, pp. 4240-4246, Jun. 2008.

4. V.N. Panteleev et al., "Sudies of uranium carbide targets of a high density," Nucl. Instr. and Meth. B, vol. 266, pp. 4247-4251, Jun. 2008.

5. V. Chudakov et al., "Abstracts of Papers", presented at $7^{\text {th }}$ Int. Conf. on Isotopes, Moscow, Sept. 4-8, 2011.

6. V. N. Panteleev et al., "Production of Cs and Fr isotopes from a high-density UC targets with different grain dimensions" Eur. Phys. A, vol. 42, pp. 495-501, Jun. 2009. 